\title{
ON OPEN AND DISCRETE MAPPINGS WITH A MODULUS CONDITION
}

\author{
Evgeny Sevost'yanov \\ Zhitomir Ivan Franko State University \\ 40 Bol'shaya Berdichevskaya Str., 10008 Zhitomir, Ukraine; esevostyanov2009@mail.ru
}

\begin{abstract}
It is proved that sense preserving continuous mappings $f: D \rightarrow \mathbf{R}^{n}$ of a domain $D$ in $\mathbf{R}^{n}, n \geqslant 2$, satisfying some general inequalities for $p$-modulus of families of curves are open and discrete.
\end{abstract}

\section{Preliminaries}

The paper is devoted to the study of quasiregular mappings and their natural generalizations investigated long time, see e.g. [AC, $\mathrm{Cr}_{1}, \mathrm{Cr}_{2}, \mathrm{Gol}_{1}, \mathrm{Gol}_{2}$, GRSY, HK, IM, KO, MRV, MRSY, Re, Ri, UV] and further references therein.

Let us give some definitions. Everywhere further $D$ is a domain in $\mathbf{R}^{n}, n \geq 2$, $m$ is the Lebesgue measure in $\mathbf{R}^{n}, m(A)$ the Lebesgue measure of a set $A \subset \mathbf{R}^{n}$. A mapping $f: D \rightarrow \mathbf{R}^{n}$ is called discrete if $f^{-1}(y)$ consists of isolated points for each $y \in \mathbf{R}^{n}$, and $f$ is said to be open if it maps open sets onto open sets. The notation $f: D \rightarrow \mathbf{R}^{n}$ assumes that $f$ is continuous. A mapping $f$ is said to be sensepreserving if the topological index $\mu(y, f, G)>0$ for an arbitrary domain $G \subset D$ such that $\bar{G} \subset D$ and $y \in f(G) \backslash f(\partial G)$, see e.g. [Re, II.2]. Given a mapping $f: D \rightarrow \mathbf{R}^{n}$, a set $E \subset D$ and a point $y \in \mathbf{R}^{n}$, we define the multiplicity function $N(y, f, E)$ as the number of pre-images of $y$ in $E$, i.e.,

$$
N(y, f, E)=\operatorname{card}\{x \in E: f(x)=y\}
$$

and

$$
N(f, E)=\sup _{y \in \mathbf{R}^{n}} N(y, f, E) .
$$

A set $H \subset \overline{\mathbf{R}^{n}}$ is called totally disconnected if its every component degenerates to a point; in this case we write $\operatorname{dim} H=0$ where dim denotes the topological dimension of $H$ (see [HW, Section 1, Ch. II]). A mapping $f: D \rightarrow \overline{\mathbf{R}^{n}}$ is said to be light if $\operatorname{dim}\left\{f^{-1}(y)\right\}=0$ for every $y \in \overline{\mathbf{R}^{n}}$. Set

$$
B\left(x_{0}, r\right)=\left\{x \in \mathbf{R}^{n}:\left|x-x_{0}\right|<r\right\}, \quad \mathbf{B}^{n}:=B(0,1), \quad \mathbf{S}^{n-1}:=S(0,1),
$$

$\Omega_{n}$ is a volume of the unit ball $\mathbf{B}^{n}$ in $\mathbf{R}^{n}$, and $\omega_{n-1}$ is an area of the unit sphere $\mathbf{S}^{n-1}$ in $\mathbf{R}^{n}$.

A curve $\gamma$ in $\mathbf{R}^{n}$ is a continuous mapping $\gamma: \Delta \rightarrow \mathbf{R}^{n}$ where $\Delta$ is an interval in R. Its locus $\gamma(\Delta)$ is denoted by $|\gamma|$. Given a family $\Gamma$ of curves $\gamma$ in $\mathbf{R}^{n}$, a Borel function $\rho: \mathbf{R}^{n} \rightarrow[0, \infty]$ is called admissible for $\Gamma$, abbr. $\rho \in \operatorname{adm} \Gamma$, if

$$
\int_{\gamma} \rho(x)|d x| \geq 1
$$

doi:10.5186/aasfm.2016.4105

2010 Mathematics Subject Classification: Primary 30C65; Secondary 30C62.

Key words: Mappings with bounded and finite distortion, moduli of families of curves, discrete and open mappings. 
for each (locally rectifiable) $\gamma \in \Gamma$. Given $p \geq 1$, the $p$-modulus of $\Gamma$ is defined by

$$
M_{p}(\Gamma):=\inf _{\rho \in \operatorname{adm} \Gamma} \int_{\mathbf{R}^{n}} \rho^{p}(x) d m(x)
$$

interpreted as $+\infty$ if $\operatorname{adm} \Gamma=\varnothing$. Note that $M_{p}(\varnothing)=0 ; M_{p}\left(\Gamma_{1}\right) \leq M_{p}\left(\Gamma_{2}\right)$ whenever $\Gamma_{1} \subset \Gamma_{2}$, and $M_{p}\left(\bigcup_{i=1}^{\infty} \Gamma_{i}\right) \leq \sum_{i=1}^{\infty} M_{p}\left(\Gamma_{i}\right)$, see [Va, Theorem 6.2].

Denote $\Gamma(E, F, D)$ a family of all paths $\gamma:[a, b] \rightarrow \overline{\mathbf{R}^{n}}$, which join sets $E$ and $F$ in $D$, i.e., $\gamma(a) \in E, \gamma(b) \in F$ and $\gamma(t) \in D$ for $t \in(a, b)$.

The following fact has been established in [Sev]. Let $f$ be a sense-preserving mapping of a domain $D \subset \mathbf{R}^{n}, n \geqslant 2$, into $\mathbf{R}^{n}$ obeying a condition

$$
M(\Gamma) \leqslant \int_{f(D)} Q(y) \cdot \rho_{*}^{n}(y) d m(y)
$$

for every $\rho_{*} \in \operatorname{adm} f(\Gamma)$ with respect to the conformal modulus $M(\Gamma):=M_{n}(\Gamma)$ and a given function $Q: \mathbf{R}^{n} \rightarrow[0, \infty]$. Then $f$ is open and discrete whenever $Q$ satisfies some conditions. Given $y_{0} \in f(D)$ and numbers $0<r_{1}<r_{2}<\infty$, we denote

$$
A\left(r_{1}, r_{2}, y_{0}\right)=\left\{y \in \mathbf{R}^{n}: r_{1}<\left|y-y_{0}\right|<r_{2}\right\} .
$$

The goal of the present paper is to prove a similar result for $n-1<p \leqslant n$. Namely, given $y_{0} \in f(D)$ and $0<r_{1}<r_{2}<\infty$, let $\Gamma\left(y_{0}, r_{1}, r_{2}\right)$ be the family of all paths $\gamma$ in $D$ such that $f(\gamma) \in \Gamma\left(S\left(y_{0}, r_{1}\right), S\left(y_{0}, r_{2}\right), A\left(r_{1}, r_{2}, y_{0}\right)\right)$. Instead of (1.1), assume that $f$ satisfies the inequality

$$
M_{p}\left(\Gamma\left(y_{0}, r_{1}, r_{2}\right)\right) \leqslant \int_{f(D)} Q(y) \cdot \eta^{p}\left(\left|y-y_{0}\right|\right) d m(y)
$$

for some $p \in(n-1, n]$, every $y_{0} \in f(D)$, any $0<r_{1}<r_{2}<\infty$, and any nonnegative Lebesgue measurable function $\eta:\left(r_{1}, r_{2}\right) \rightarrow[0, \infty]$ with

$$
\int_{r_{1}}^{r_{2}} \eta(r) d r \geqslant 1
$$

Observe that the inequality (1.3) is much weaker than (1.1) even for $p=n$. In fact, let $\rho_{*} \in \operatorname{adm} f(\Gamma)$, and assume that the relation (1.1) holds. We show that the inequality (1.3) is true. To this end, pick arbitrary $y_{0} \in f(D)$ and set $\rho_{*}(y):=\eta\left(\left|y-y_{0}\right|\right)$, where $\eta$ satisfies (1.4). Note that $\rho_{*} \in \Gamma\left(S_{1}, S_{2}, A\right)$ because $\int_{\gamma} \rho_{*}(y)|d y| \geqslant \int_{r_{1}}^{r_{2}} \eta(t) d t \geqslant 1$ for every $\gamma \in \Gamma\left(S_{1}, S_{2}, A\right)$ (cf. [Va, theorem 5.7]). Thus, the inequality (1.1) becomes (1.3).

The present paper is devoted to the study of the following question:

What are the properties of the majorant $Q$ which ensure for mappings $f$ obeying (1.3) for some $n-1<p \leqslant n$ to be discrete and open?

Following [IR], we say that a function $\varphi: \mathbf{R}^{n} \rightarrow \mathbf{R}$ has a finite mean oscillation at a point $x_{0} \in \mathbf{R}^{n}$, write $\varphi \in F M O\left(x_{0}\right)$, if

$$
\varlimsup_{\varepsilon \rightarrow 0} \frac{1}{\Omega_{n} \varepsilon^{n}} \int_{B\left(x_{0}, \varepsilon\right)}\left|\varphi(x)-\varphi_{\varepsilon}\right| d m(x)<\infty,
$$

where $\Omega_{n}$ is the volume of the unit ball in $\mathbf{R}^{n}$ and

$$
\varphi_{\varepsilon}=\frac{1}{\Omega_{n} \varepsilon^{n}} \int_{B\left(x_{0}, \varepsilon\right)} \varphi(x) d m(x) .
$$


Given a Lebesgue measurable function $Q: \mathbf{R}^{n} \rightarrow[0, \infty], q_{x_{0}}(r)$ denotes the integral average of $Q(x)$ over the sphere $S\left(x_{0}, r\right)$, i.e.

$$
q_{x_{0}}(r):=\frac{1}{\omega_{n-1} r^{n-1}} \int_{\left|x-x_{0}\right|=r} Q(x) d S
$$

where $d S$ is an area element of $S$.

The main result of the present paper is the following

Theorem 1.1. Let $p \in(n-1, n]$, and $Q: \mathbf{R}^{n} \rightarrow(0, \infty)$ be a Lebesgue measurable function. Assume that $f: D \rightarrow \mathbf{R}^{n}$ is a sense-preserving mapping satisfying (1.3) for every $y_{0} \in f(D)$, any $0<r_{1}<r_{2}<\infty$, and any nonnegative Lebesgue measurable function $\eta:\left(r_{1}, r_{2}\right) \rightarrow[0, \infty]$ obeying (1.4). Then $f$ is discrete and open whenever the function $Q$ satisfies at least one of the following conditions:

1) $Q \in F M O\left(y_{0}\right)$ for every $y_{0} \in f(D)$,

2) $q_{y_{0}}(r)=O\left(\left[\log \frac{1}{r}\right]^{n-1}\right)$ as $r \rightarrow 0$ for every $y_{0} \in f(D)$,

3) for every $y_{0} \in f(D)$ there exists $\delta\left(y_{0}\right)>0$ such that for every sufficiently small $\varepsilon>0$

$$
\int_{\varepsilon}^{\delta\left(y_{0}\right)} \frac{d t}{t^{\frac{n-1}{p-1}} q_{y_{0}}^{\frac{1}{p-1}}(t)}<\infty, \quad \int_{0}^{\delta\left(y_{0}\right)} \frac{d t}{t^{\frac{n-1}{p-1}} q_{y_{0}}^{\frac{1}{p-1}}(t)}=\infty .
$$

Remark 1.1. Theorem 1.1 can be extended to the mappings $f: D \rightarrow \overline{\mathbf{R}^{n}}$. In this case, for $y=\infty$, we must require that the conditions 1)-3) hold for $\widetilde{Q}=Q \circ \varphi$ at 0 , where $\varphi(y)=\frac{y}{|y|^{2}}, \varphi(0):=\infty$.

\section{Main lemma}

A connected compactum $C \subset \overline{\mathbf{R}^{n}}$ is called a continuum. We say that a family of paths $\Gamma_{1}$ is minorized by a family $\Gamma_{2}$, write $\Gamma_{1}>\Gamma_{2}$, if for every $\gamma \in \Gamma_{1}$ there exists a subpath which belongs to $\Gamma_{2}$. In this case, $M_{p}\left(\Gamma_{1}\right) \leqslant M_{p}\left(\Gamma_{2}\right)$ (see [Va, Theorem 6.4]).

Let $(X, \mu)$ be a metric space with measure $\mu$. For each real number $n \geq 1$, we define the Loewner function $\phi_{n}:(0, \infty) \rightarrow[0, \infty)$ on $X$ as

$$
\phi_{n}(t)=\inf \left\{M_{n}(\Gamma(E, F, X)): \Delta(E, F) \leqslant t\right\},
$$

where the infimum is taken over all disjoint nondegenerate continua $E$ and $F$ in $X$ and

$$
\Delta(E, F):=\frac{\operatorname{dist}(E, F)}{\min \{\operatorname{diam} E, \operatorname{diam} F\}} .
$$

A pathwise connected metric measure space $(X, \mu)$ is said to be a Loewner space of exponent $n$, or an $n$-Loewner space, if the Loewner function $\phi_{n}(t)$ is positive for all $t>0$ (see [MRSY, Section 2.5] or [He, Ch. 8]). Observe that $\mathbf{R}^{n}$ and $\mathbf{B}^{n} \subset \mathbf{R}^{n}$ are Loewner spaces (see [He, Theorem 8.2 and Example 8.24(a)]). As known, a condition $\mu\left(B\left(x_{0}, r\right)\right) \geqslant C \cdot r^{n}$ holds in Loewner spaces $X$ for a constant $C>0$, every point $x_{0} \in X$ and all $r<\operatorname{diam} X$. A space $X$ is called geodesic if every pair of points in $X$ can be joined by a curve whose length is equal to the distance between the points. In particular, $\mathbf{B}^{n}$ is a geodesic space. A following definition can be found in [He, Section 1.4, Ch. I] or [AS, Section 1]. A measure $\mu$ in a metric space is called doubling if all balls have finite and positive measure and there is a constant $C \geqslant 1$ such that $\mu\left(B\left(x_{0}, 2 r\right)\right) \leq C \cdot \mu\left(B\left(x_{0}, r\right)\right)$ for every $x_{0} \in X$ and all $r>0$. We also call a metric measure space $(X, \mu)$ doubling if $\mu$ is a doubling measure. Following [He, Section 7.22], given a real-valued function $u$ in a metric space $X$, a 
Borel function $\rho: X \rightarrow[0, \infty]$ is said to be an upper gradient of a function $u: X \rightarrow \mathbf{R}$ if $|u(x)-u(y)| \leqslant \int_{\gamma} \rho|d x|$ for each rectifiable curve $\gamma$ joining $x$ and $y$ in $X$. Let $(X, \mu)$ be a metric measure space and let $1 \leqslant p<\infty$. We say that $X$ admits $a$ $(1 ; p)$-Poincare inequality if there is a constant $C \geqslant 1$ such that

$$
\frac{1}{\mu(B)} \int_{B}\left|u-u_{B}\right| d \mu(x) \leqslant C \cdot(\operatorname{diam} B)\left(\frac{1}{\mu(B)} \int_{B} \rho^{p} d \mu(x)\right)^{1 / p}
$$

for all balls $B$ in $X$, for all bounded continuous functions $u$ on $B$, and for all upper gradients $\rho$ of $u$. Metric measure spaces where the inequalities

$$
\frac{1}{C} R^{n} \leqslant \mu\left(B\left(x_{0}, R\right)\right) \leqslant C R^{n}
$$

hold for a constant $C \geqslant 1$, every $x_{0} \in X$ and all $R<$ diam $X$, are called Ahlfors $n$-regular.

We need the following statement.

Proposition 2.1. The unit ball $\mathbf{B}^{n}$ is an Ahlfors $n$-regular metric space in which $(1 ; p)$-Poincare inequality holds. Moreover, the estimate

$$
M_{p}\left(\Gamma\left(E, F, \mathbf{B}^{n}\right)\right)>0
$$

holds for any continua $E, F \subset \mathbf{B}^{n}$ and every $p \in(n-1, n]$.

Proof. By comments given above, the unit ball $\mathbf{B}^{n}$ is Ahlfors $n$-regular, moreover, the space $\mathbf{B}^{n}$ is geodesic and and also a Loewner space. By [He, Theorems 9.8 and 9.5], the $(1 ; p)$-Poincare inequality holds in $\mathbf{B}^{n}$. Thus, $(2.1)$ holds by [AS, Corollary 4.8].

Let $A\left(\varepsilon, \varepsilon_{0}, y_{0}\right)$ be defined by (1.2) with $r_{1}=\varepsilon$ and $r_{2}=\varepsilon_{0}$. The following lemma provides the main tool for establishing openness and discreteness in the most general situation.

Lemma 2.1. Let $p \in(n-1, n]$, and $Q: \mathbf{R}^{n} \rightarrow(0, \infty)$ be a Lebesgue measurable function. Assume that $f: D \rightarrow \mathbf{R}^{n}$ is a sense-preserving mapping satisfying (1.3) for every $y_{0} \in f(D)$, any $0<r_{1}<r_{2}<\infty$, and any nonnegative Lebesgue measurable function $\eta:\left(r_{1}, r_{2}\right) \rightarrow[0, \infty]$ obeying (1.4). Suppose that, in addition, for every $y_{0} \in f(D)$ and some $\varepsilon_{0}>0$,

$$
\int_{A\left(\varepsilon, \varepsilon_{0}, y_{0}\right)} Q(y) \cdot \psi^{p}\left(\left|y-y_{0}\right|\right) d m(y)=o\left(I^{p}\left(\varepsilon, \varepsilon_{0}\right)\right) \quad \text { as } \varepsilon \rightarrow 0
$$

where $\psi(t):(0, \infty) \rightarrow[0, \infty]$ is a nonnegative Lebesgue measurable function such that

$$
0<I\left(\varepsilon, \varepsilon_{0}\right):=\int_{\varepsilon}^{\varepsilon_{0}} \psi(t) d t<\infty \quad \forall \varepsilon \in\left(0, \varepsilon_{0}\right) .
$$

Then $f$ is open and discrete.

Remark 2.1. In Lemma 2.1, we may assume only that $\int_{\varepsilon}^{\varepsilon_{0}} \psi(t) d t>0$ for some $\varepsilon$ and $\varepsilon_{*} \in\left(0, \varepsilon_{0}\right)$ instead of the condition $I\left(\varepsilon, \varepsilon_{0}\right)>0$ for all $\varepsilon \in\left(0, \varepsilon_{0}\right)$. Note also that the integral (2.2) is increasing under decreasing $\varepsilon$. Thus, since $Q(x)>0$, $I\left(\varepsilon, \varepsilon_{0}\right) \rightarrow \infty$ as $\varepsilon \rightarrow 0$ follows from (2.2) and (2.3).

Proof of Lemma 2.1. Without any loss of generality, we may asssume that $D=\mathbf{B}^{n}$. Since every light sense-preserving mapping $f: D \rightarrow \mathbf{R}^{n}$ is open and discrete in $D$, see e.g., [TY, Corollary, p. 333], it is sufficient to prove that $f$ is light. Let us assume the contrary. Then there exists $y_{0} \in \mathbf{R}^{n}$ such that the set $\left\{f^{-1}\left(y_{0}\right)\right\}$ is not 
totally disconnected, i.e., there exists a nondegenerate continuum $C \subseteq\left\{f^{-1}\left(y_{0}\right)\right\}$. Since $f$ is sense-preserving, $f \not \equiv y_{0}$. Then by the continuity of $f$ there exist $x_{0} \in D$ and $\delta_{0}>0$ such that $\overline{B\left(x_{0}, \delta_{0}\right)} \subset D$ and

$$
f(x) \neq y_{0} \quad \forall x \in \overline{B\left(x_{0}, \delta_{0}\right)} .
$$

By [Na, Lemma 1.15] for $p=n$, and Proposition 2.1 for $p \in(n-1, n)$,

$$
M_{p}\left(\Gamma\left(C, \overline{B\left(x_{0}, \delta_{0}\right)}, \mathbf{B}^{n}\right)\right)>0 .
$$

By (2.4), since $f(C)=\left\{y_{0}\right\}$, every path of $\Delta=f\left(\Gamma\left(C, \overline{B\left(x_{0}, \delta_{0}\right)}, \mathbf{B}^{n}\right)\right)$ does not degenerate to a point. On the other hand, one of the endpoints of every path of $\Delta$ is $y_{0}$. Let $\Gamma_{i}$ be a family of all paths $\alpha_{i}(t):[0,1] \rightarrow \mathbf{R}^{n}$ such that $\alpha_{i}(0)=y_{0}$ and $\alpha_{i}(1) \in S\left(y_{0}, r_{i}\right), r_{i} \in\left(0, \varepsilon_{0}\right), i=1,2, \ldots, r_{i} \rightarrow 0$ as $i \rightarrow \infty$. Note that

$$
\Gamma\left(C, \overline{B\left(x_{0}, \delta_{0}\right)}, \mathbf{B}^{n}\right)=\bigcup_{i=1}^{\infty} \Gamma_{i}^{*}
$$

where $\Gamma_{i}^{*}$ is the family of all paths $\gamma$ in $\Gamma\left(C, \overline{B\left(x_{0}, \delta_{0}\right)}, \mathbf{B}^{n}\right)$ such that $f(\gamma)$ has a subpath in $\Gamma_{i}$. Observe that

$$
\Gamma_{i}^{*}>\Gamma\left(\varepsilon, r_{i}, y_{0}\right)
$$

for every $\varepsilon \in\left(0, r_{i}\right)$ where $\Gamma\left(\varepsilon, r_{i}, y_{0}\right)$ is the family of all paths $\gamma$ in $D$ such that $f(\gamma) \in \Gamma\left(S\left(y_{0}, \varepsilon\right), S\left(y_{0}, r_{i}\right), A\left(\varepsilon, r_{i}, y_{0}\right)\right)$. Set

$$
\eta_{i, \varepsilon}(t)= \begin{cases}\psi(t) / I\left(\varepsilon, r_{i}\right), & t \in\left(\varepsilon, r_{i}\right), \\ 0, & t \notin\left(\varepsilon, r_{i}\right),\end{cases}
$$

where $I\left(\varepsilon, r_{i}\right)=\int_{\varepsilon}^{r_{i}} \psi(t) d t$. Observe that $\int_{\varepsilon}^{r_{i}} \eta_{i, \varepsilon}(t) d t=1$. Now we can apply (1.3). By (1.3) and (2.7),

$$
M_{p}\left(\Gamma_{i}^{*}\right) \leqslant M_{p}\left(\Gamma\left(r_{i}, \varepsilon, y_{0}\right)\right) \leqslant \int_{A\left(\varepsilon, \varepsilon_{0}, y_{0}\right)} Q(y) \cdot \eta_{i, \varepsilon}^{p}\left(\left|y-y_{0}\right|\right) d m(y) \leqslant \mathfrak{F}_{i}(\varepsilon)
$$

where $\mathfrak{F}_{i}(\varepsilon)=\frac{1}{I\left(\varepsilon, r_{i}\right)^{p}} \int_{A\left(\varepsilon, \varepsilon_{0}, y_{0}\right)} Q(y) \psi^{p}\left(\left|y-y_{0}\right|\right) d m(y)$ and $I\left(\varepsilon, r_{i}\right)=\int_{\varepsilon}^{r_{i}} \psi(t) d t$. By $(2.2)$,

$$
\int_{A\left(\varepsilon, \varepsilon_{0}, y_{0}\right)} Q(y) \psi^{p}\left(\left|y-y_{0}\right|\right) d m(y)=G(\varepsilon) \cdot\left(\int_{\varepsilon}^{\varepsilon_{0}} \psi(t) d t\right)^{p},
$$

where $G(\varepsilon) \rightarrow 0$ as $\varepsilon \rightarrow 0$ by the assumptions of the lemma. Note that $\mathfrak{F}_{i}(\varepsilon)=$ $G(\varepsilon) \cdot\left(1+\frac{\int_{r_{i}}^{\varepsilon_{0}} \psi(t) d t}{\int_{\varepsilon}^{r_{i}} \psi(t) d t}\right)^{p}$, where $\int_{r_{i}}^{\varepsilon_{0}} \psi(t) d t<\infty$ and $\int_{\varepsilon}^{r_{i}} \psi(t) d t \rightarrow \infty$ as $\varepsilon \rightarrow 0$, because the left-hand side in (2.2) is increasing under decreasing $\varepsilon$. Thus, $\mathfrak{F}_{i}(\varepsilon) \rightarrow 0$ as $\varepsilon \rightarrow 0$, and by $(2.8) M_{p}\left(\Gamma_{i}^{*}\right)=0$ for all $i$. Finally, by (2.6) and the inequality $M_{p}\left(\bigcup_{i=1}^{\infty} \Gamma_{i}^{*}\right) \leqslant$ $\sum_{i=1}^{\infty} M_{p}\left(\Gamma_{i}^{*}\right)$ [Va, Theorem 6.2], we obtain that $M_{p}\left(\Gamma\left(C, \overline{B\left(x_{0}, \delta_{0}\right)}, \mathbf{B}^{n}\right)\right)=0$, which contradicts to (2.5). Thus, $f$ is light and, therefore, $f$ is open and discrete (see [TY, Corollary, p. 333]). 


\section{Proof of the main result}

In this section we show that the assertion of Theorem 1.1 follows from Lemma 2.1. For this goal, given $Q \in F M O\left(y_{0}\right)$, we may apply the function $\psi(t)=\left(t \log \frac{1}{t}\right)^{-n / p}$. Indeed, by [IR, Corollary 2.3], see also [MRSY, Corollary 6.3, Ch. 6], we obtain

$$
\int_{\varepsilon<\left|y-y_{0}\right|<\varepsilon_{0}} Q(y) \cdot \psi^{p}\left(\left|y-y_{0}\right|\right) d m(y)=O\left(\log \log \frac{1}{\varepsilon}\right) \quad \text { as } \varepsilon \rightarrow 0
$$

and

$$
I\left(\varepsilon, \varepsilon_{0}\right)=\int_{\varepsilon}^{\varepsilon_{0}} \psi(t) d t>\log \frac{\log \frac{1}{\varepsilon}}{\log \frac{1}{\varepsilon_{0}}}>\log \log \frac{1}{\varepsilon}
$$

for sufficiently small $\varepsilon_{0}>0$, and hence

$$
\frac{1}{I^{p}\left(\varepsilon, \varepsilon_{0}\right)} \int_{\varepsilon<\left|y-y_{0}\right|<\varepsilon_{0}} Q(y) \cdot \psi^{p}\left(\left|y-y_{0}\right|\right) d m(y) \leqslant C\left(\log \log \frac{1}{\varepsilon}\right)^{1-p} \rightarrow 0 \quad \text { as } \varepsilon \rightarrow 0 .
$$

The latter yields the desired conclusion for the case 1) because (2.2)-(2.3) hold.

Note that the case 2) is a consequence of case 3) and therefore, we may restrict ourselves by checking the condition 3 ). For this case we pick the function

$$
\psi(t)= \begin{cases}1 /\left[t^{\frac{n-1}{p-1}} q_{y_{0}}^{\frac{1}{p-1}}(t)\right], & t \in\left(\varepsilon, \varepsilon_{0}\right) \\ 0, & t \notin\left(\varepsilon, \varepsilon_{0}\right)\end{cases}
$$

in Lemma 2.1 and thus,

$$
I\left(\varepsilon, \varepsilon_{0}\right)=\int_{\varepsilon}^{\varepsilon_{0}} \frac{d r}{r^{\frac{n-1}{p-1}} q_{y_{0}}^{\frac{1}{p-1}}(r)} .
$$

Applying the Fubini theorem ([Sa, Theorem 8.1, Ch. III]), we obtain

$$
\int_{\varepsilon<\left|y-y_{0}\right|<\varepsilon_{0}} Q(y) \cdot \psi^{p}\left(\left|y-y_{0}\right|\right) d m(y)=\omega_{n-1} \cdot I\left(\varepsilon, \varepsilon_{0}\right)=o\left(I^{p}\left(\varepsilon, \varepsilon_{0}\right)\right) \quad \text { as } \varepsilon \rightarrow 0,
$$

where $\omega_{n-1}$ denotes the area of the unit sphere $\mathbf{S}^{n-1}$ in $\mathbf{R}^{n}$.

\section{Corollaries}

The following statements can be derived from Lemma 2.1.

Corollary 4.1. Let $p \in(n-1, n]$, and $Q: \mathbf{R}^{n} \rightarrow(0, \infty)$ be a Lebesgue measurable function. Assume that $f: D \rightarrow \mathbf{R}^{n}$ is a sense-preserving mapping satisfying (1.3) for every $y_{0} \in f(D)$, any $0<r_{1}<r_{2}<\infty$, and any nonnegative Lebesgue measurable function $\eta:\left(r_{1}, r_{2}\right) \rightarrow[0, \infty]$ obeying (1.4). Then $f$ is discrete and open whenever the function $Q$ satisfies the both conditions:

1) $\int_{\varepsilon}^{\delta_{0}} \frac{d t}{t q_{y_{0}}^{\frac{1}{n-1}}(t)}<\infty$ for every $y_{0} \in f(D)$, some $\delta_{0}=\delta_{0}\left(y_{0}\right)$ and sufficiently small $\varepsilon>0$,

2) $\int_{0}^{\delta_{0}} \frac{d t}{t q_{y_{0}}^{\frac{1}{n-1}}(t)}=\infty$ for every $y_{0} \in f(D)$ and some $\delta_{0}=\delta_{0}\left(y_{0}\right)$. 
Proof. Arguing similarly to the proof of the case 3) in Theorem 1.1 and choosing

$$
\psi(t)= \begin{cases}\left(1 /\left[t q_{y_{0}}^{\frac{1}{n-1}}(t)\right]\right)^{n / p}, & t \in\left(\varepsilon, \varepsilon_{0}\right), \\ 0, & t \notin\left(\varepsilon, \varepsilon_{0}\right),\end{cases}
$$

in Lemma 2.1, we obtain the desired conclusion.

Corollary 4.2. Let $p \in(n-1, n)$, and $Q: \mathbf{R}^{n} \rightarrow(0, \infty)$ be a Lebesgue measurable function. Assume that $f: D \rightarrow \mathbf{R}^{n}$ is a sense-preserving mapping satisfying (1.3) for every $y_{0} \in f(D)$, any $0<r_{1}<r_{2}<\infty$, and any nonnegative Lebesgue measurable function $\eta:\left(r_{1}, r_{2}\right) \rightarrow[0, \infty]$ obeying (1.4). Then $f$ is discrete and open whenever $Q \in L_{\text {loc }}^{s}$ for some $s \geqslant n /(n-p)$.

Proof. Given $\varepsilon_{0} \in(0, \infty)$ and $x_{0} \in D$, set $G:=B\left(x_{0}, \varepsilon_{0}\right)$. Note that the function $\psi(t):=1 / t$ satisfies (2.3). Thus, in order to apply Lemma 2.1 it remains to verify (2.2). Indeed, by the Hölder inequality we obtain

$$
\begin{aligned}
& \int_{\varepsilon<\left|x-x_{0}\right|<\varepsilon_{0}} \frac{Q(x)}{\left|x-x_{0}\right|^{p}} d m(x) \\
& \leqslant\left(\int_{\varepsilon<\left|x-x_{0}\right|<\varepsilon_{0}} \frac{1}{\left|x-x_{0}\right|^{p q}} d m(x)\right)^{\frac{1}{q}}\left(\int_{G} Q^{q^{\prime}}(x) d m(x)\right)^{\frac{1}{q^{\prime}}}
\end{aligned}
$$

where $q=n / p, 1 / q+1 / q^{\prime}=1$, i.e., $q^{\prime}=n /(n-p)$. Observe that the first integral in the right-hand side of (4.1) can be implicitly calculated. Namely, by the Fubini theorem

$$
\int_{\varepsilon<\left|x-x_{0}\right|<\varepsilon_{0}} \frac{1}{\left|x-x_{0}\right|^{p q}} d m(x)=\omega_{n-1} \int_{\varepsilon}^{\varepsilon_{0}} \frac{d t}{t}=\omega_{n-1} \log \frac{\varepsilon_{0}}{\varepsilon} .
$$

Following notation of Lemma 2.1, we have

$\frac{1}{I^{p}\left(\varepsilon, \varepsilon_{0}\right)} \int_{\varepsilon<\left|x-x_{0}\right|<\varepsilon_{0}} \frac{Q(x)}{\left|x-x_{0}\right|^{p}} d m(x) \leqslant \omega_{n-1}^{\frac{p}{n}}\|Q\|_{L^{\frac{n}{n-p}}(G)}\left(\log \frac{\varepsilon_{0}}{\varepsilon}\right)^{-p+\frac{p}{n}} \rightarrow 0$ as $\varepsilon \rightarrow 0$

that implies (2.2). Thus, the desired conclusion follows from Lemma 2.1.

\section{Examples}

First of all, let us give some examples of mappings obeying (1.1) and (1.3). It is known that, for an arbitrary quasiregular mapping $f: D \rightarrow \mathbf{R}^{n}$, one has

$$
M(\Gamma) \leq N(f, A) K_{O}(f) M(f(\Gamma))
$$

for a constant $K_{O}(f) \geqslant 1$, for any Borel set $A$ in the domain $D$ such that $N(f, A)<\infty$ and any family $\Gamma$ of curves $\gamma$ in $A$ (see [MRV, Theorem 3.2] or [Ri, Theorem 6.7, Chap. II]). Thus, for any such quasiregular mapping, the inequalities (1.1) and (1.3) hold.

Let us give other examples. Set at points $x \in D$ of differentiability of $f$

$$
\left\|f^{\prime}(x)\right\|=\max _{h \in \mathbf{R}^{n} \backslash\{0\}} \frac{\left|f^{\prime}(x) h\right|}{|h|}, \quad J(x, f)=\operatorname{det} f^{\prime}(x),
$$


and define for any $x \in D$ and $p \geqslant 1$

$$
K_{O, p}(x, f)= \begin{cases}\frac{\left\|f^{\prime}(x)\right\|^{p}}{|J(x, f)|}, & J(x, f) \neq 0 \\ 1, & f^{\prime}(x)=0 \\ \infty, & \text { otherwise. }\end{cases}
$$

We say that a property $P$ holds for $p$-almost every (p-a.e.) curves $\gamma$ in a family $\Gamma$ if the subfamily of all curves in $\Gamma$, for which $P$ fails, has $p$-modulus zero. Recall that a mapping $f: D \rightarrow \mathbf{R}^{n}$ is said to have $N$-property (by Luzin) if $m(f(S))=0$ whenever $m(S)=0$ for $S \subset \mathbf{R}^{n}$. Similarly, $f$ has the $N^{-1}$-property if $m\left(f^{-1}(S)\right)=0$ whenever $m(S)=0$.

If $\gamma: \Delta \rightarrow \mathbf{R}^{n}$ is a locally rectifiable curve, then there is the unique nondecreasing length function $l_{\gamma}$ of $\Delta$ onto a length interval $\Delta_{\gamma} \subset \mathbf{R}$ with a prescribed normalization $l_{\gamma}\left(t_{0}\right)=0 \in \Delta_{\gamma}, t_{0} \in \Delta$, such that $l_{\gamma}(t)$ is equal to the length of the subcurve $\left.\gamma\right|_{\left[t_{0}, t\right]}$ of $\gamma$ if $t>t_{0}, t \in \Delta$, and $l_{\gamma}(t)$ is equal to minus length of $\left.\gamma\right|_{\left[t, t_{0}\right]}$ if $t<t_{0}, t \in \Delta$. Let $g:|\gamma| \rightarrow \mathbf{R}^{n}$ be a continuous mapping, and suppose that the curve $\widetilde{\gamma}=g \circ \gamma$ is also locally rectifiable. Then there is a unique non-decreasing function $L_{\gamma, g}: \Delta_{\gamma} \rightarrow \Delta_{\tilde{\gamma}}$ such that $L_{\gamma, g}\left(l_{\gamma}(t)\right)=l_{\widetilde{\gamma}}(t)$ for all $t \in \Delta$. We say that a mapping $f: D \rightarrow \mathbf{R}^{n}$ is absolutely continuous on paths with respect to p-modulus, write $f \in A C P_{p}$, if for $p$-a.e. curve $\gamma: \Delta \rightarrow D$ the function $L_{\gamma, f}$ is locally absolutely continuous on $\Delta$.

The following result is a generalization of the above classical inequality (5.1) for quasiregular mappings and provides an example of the inequality (1.3) (see [Ri, Theorem 2.4, Ch. II]).

Theorem 5.1. Let a mapping $f: D \rightarrow \mathbf{R}^{n}$ be differentiable a.e. in $D$, have $N$ and $N^{-1}$-properties and possess $A C P_{p}$-property for some $p \geqslant 1$. Let $A$ be a Borel set in $D$, and let $\Gamma$ be a family of paths in $A$. Suppose that $K_{O, p}(x, f) \leqslant \alpha(f(x))$ a.e. for a Borel function $\alpha: \mathbf{R}^{n} \rightarrow[0, \infty]$. Then

$$
M_{p}(\Gamma) \leq \int_{\mathbf{R}^{n}} \rho^{\prime p}(y) N(y, f, A) \alpha(y) d m(y) \quad \forall \rho^{\prime} \in \operatorname{adm} f(\Gamma) .
$$

Recall that $N(y, f, A)$ is Lebesgue measurable for any Borel measurable set $A$ (see [RR, Theorem of Section IV.1.2]).

Proof. Let $\rho^{\prime} \in \operatorname{adm} f(\Gamma)$. Set $\rho(x)=\rho^{\prime}(f(x))\left\|f^{\prime}(x)\right\|$ for $x \in A$ and $\rho(x)=0$ otherwise. Let $\Gamma_{0}$ be a family of all locally rectifiable curves of $\Gamma$ where $f$ is locally absolutely continuous. Since $f \in A C P_{p}, M_{p}(\Gamma)=M_{p}\left(\Gamma_{0}\right)$. Now, by [Ri, Lemma 2.2, Ch. II], $\int_{\gamma} \rho(x)|d x|=\int_{\gamma} \rho^{\prime}(f(x))|| f^{\prime}(x)|||d x| \geq \int_{f \circ \gamma} \rho^{\prime}(y)|d y| \geq 1$, and, consequently, $\rho \in \operatorname{adm} \Gamma_{0}$. By the change of variables formula

$$
\begin{aligned}
M_{p}(\Gamma) & =M_{p}\left(\Gamma_{0}\right) \leq \int_{\mathbf{R}^{n}} \rho^{p}(x) d m(x)=\int_{A} \frac{\rho^{\prime p}(f(x))\left\|f^{\prime}(x)\right\|^{p}|J(x, f)|}{|J(x, f)|} d m(x) \\
& \leq \int_{A} \rho^{\prime p}(f(x)) \alpha(f(x))|J(x, f)| d m(x)=\int_{\mathbf{R}^{n}} \rho^{\prime p}(y) N(y, f, A) \alpha(y) d m(y),
\end{aligned}
$$

see [MRSY, Proposition 8.3]. Here we take into account, that $J(x, f) \neq 0$ a.e., see [MRSY, Proposition 8.3]. The theorem is proved.

By Theorem 5.1 we obtain the following

Corollary 5.1. Let $f: D \rightarrow \mathbf{R}^{n}$ be differentiable a.e. in $D$, have $N$ - and $N^{-1}$ properties, and $f \in W_{\text {loc }}^{1, p}$ for some $p \geqslant 1$. Let $A$ in $D$ be a Borel set and $\Gamma$ be a family of paths in $A$. Suppose that $K_{O, p}(x, f) \leqslant \alpha(f(x))$ a.e. for a Borel function 
$\alpha: \mathbf{R}^{n} \rightarrow[0, \infty]$. Then

$$
M_{p}(\Gamma) \leq \int_{\mathbf{R}^{n}} \rho^{\prime p}(y) N(y, f, A) \alpha(y) d m(y) \quad \text { for all } \rho^{\prime} \in \operatorname{adm} f(\Gamma) .
$$

Indeed, as known, $W_{\text {loc }}^{1, p}=A C L^{p}$ (see [Maz, Theorems 1 and 2, section 1.1.3]). On the other hand, $A C L^{p} \subset A C P_{p}$ by the Fuglede lemma (see [Va, Theorem 28.2]). Thus, by Theorem 5.1 we obtain the inequality of type (1.3).

The next statement follows from Theorems 1.1 and 5.1, Corollaries 4.1 and 4.2.

Corollary 5.2. Let $p \in(n-1, n], f: D \rightarrow \mathbf{R}^{n}$ be differentiable a.e. in $D$, have $N$ - and $N^{-1}$-properties, $A C P_{p}$-property for some $p \geqslant 1$ and $K_{O, p}(x, f) \leqslant \alpha(f(x))$ a.e. for a Borel function $\alpha: \mathbf{R}^{n} \rightarrow[0, \infty]$. Let $\Gamma$ be a family of paths in a Borel set $A \subseteq D$ and let $Q(y)=N(y, f, D) \cdot \max \{\alpha(y), 1\}$. Then $f$ is open and discrete whenever $Q$ satisfies at least one of the following conditions:

1) $Q \in F M O\left(y_{0}\right)$ for every $y_{0} \in f(D)$;

2) for every $y_{0} \in f(D) q_{y_{0}}(r)=O\left(\left[\log \frac{1}{r}\right]^{n-1}\right)$ as $r \rightarrow 0$, where $q_{y_{0}}(r)$ is defined in (1.5);

3) for all $y_{0} \in f(D)$ there exists $\delta>0$ : for all $\varepsilon \in(0, \delta)$ : $\int_{\varepsilon}^{\delta} \frac{d t}{t^{\frac{n-1}{p-1}} q_{y_{0}}^{\frac{1}{p-1}}(t)}<\infty$ and $\int_{0}^{\delta} \frac{d t}{t^{\frac{n-1}{p-1}} q_{y_{0}}^{\frac{1}{p-1}}(t)}=\infty$

4) for all $y_{0} \in f(D)$ there exists $\delta>0$ : for all $\varepsilon \in(0, \delta)$ : $\int_{\varepsilon}^{\delta} \frac{d t}{t q_{y_{0}}^{\frac{1}{n-1}}(t)}<\infty$ and $\int_{0}^{\delta} \frac{d t}{t q_{y_{0}}^{\frac{1}{n-1}}(t)}=\infty$

5) $p \in(n-1, n)$ and $Q \in L_{\text {loc }}^{s}\left(\mathbf{R}^{n}\right)$ for some $s \geqslant \frac{n}{n-p}$.

In particular, the assertion of Corollary 5.2 holds if $f \in W_{\text {loc }}^{1, p}$ instead of $f \in A C P_{p}$.

Note that the preserving orientation is essential condition for mappings $f$ in all statements given above. An example of a mapping $f$ with finite length distortion that does not preserve orientation and such that $M(f(\Gamma))=M(\Gamma)$, i.e., $Q \equiv 1$, but is neither discrete nor open, was given in [MRSY, Section 8.10].

We also give another simple example which shows that the preserving orientation can not be dropped. Let $x=\left(x_{1}, \ldots, x_{n}\right)$. We define $f$ as the identical mapping in the closed domain $\left\{x_{n} \geqslant 0\right\}$ and set $f(x)=\left(x_{1}, \ldots,-x_{n}\right)$ for $x_{n}<0$. Note that the mapping $f$ preserves the lengths of paths. Therefore, $f$ satisfies the inequality (1.3) with $Q \equiv 1$. This mapping is discrete but not open. In fact, under the mapping $f$ the ball $\mathbf{B}^{n}$ is mapped onto the set $\left\{y=\left(y_{1}, \ldots, y_{n}\right) \in \mathbf{R}^{n}:|y|<1, y_{n} \geqslant 0\right\}$ which is not open in $\mathbf{R}^{n}$.

Remark 5.1. Results obtained in the paper can be applied to various classes of plane and space mappings (see e.g. [GRSY] and [MRSY]).

Note that we discuss the case while $p$ ranges between $n-1$ and $n$. The question on discreteness and openness of the mappings obeying the same modulus conditions for $1 \leq p \leq n-1$ remains open. 


\section{References}

[AS] Adamowicz, T., and N. Shanmugalingam: Non-conformal Loewner type estimates for modulus of curve families. - Ann. Acad. Sci. Fenn. Math. 35, 2010, 609-626.

[AC] Andreian Cazacu, C.: On the length-area dilatation. - Complex Var. Theory Appl. 50, 2005, 765-776.

[Cr 1 CristeA, M.: Local homeomorphisms having local $A C L^{n}$ inverses. - Complex Var. Elliptic Equ. 53:1, 2008, 77-99.

[Cr $\mathrm{Cr}_{2}$ CRisteA, M.: Open discrete mappings having local $A C L^{n}$ inverses. - Complex Var. Elliptic Equ. 55:1-3, 2010, 61-90.

[Gol 1 Golberg, A.: Homeomorphisms with finite mean dilatations. - Contemp. Math. 382, 2005, 177-186.

[Gol $\left.\mathrm{G}_{2}\right]$ Golberg, A.: Differential properties of $(\alpha, Q)$-homeomorphisms. - In: Further Progress in Analysis, World Scientific Publ., 2009, 218-228.

[GRSY] Gutlyanskit, V. Ya., V. I. Ryazanov, U. Srebro, and E. Yakubov: The Beltrami equation: A geometric approach. - Dev. Math. 26, New York etc., Springer, 2012.

[He] Heinonen, J.: Lectures on analysis on metric spaces. - Springer, New York, 2001.

[HK] Hencl, S., and P. Koskela: Lectures on mappings of finite distortion. - Lecture Notes in Math. 2096, Springer, 2014.

[HW] Hurewicz, W., and H. Wallman: Dimension theory. - Princeton Univ. Press, 1948.

[IR] Ignat'Ev, A., and V. RyazAnov: Finite mean oscillation in the mapping theory. - Ukr. Math. Bull. 2:3, 2005, 403-424.

[IM] Iwaniec, T., and G. Martin: Geometric function theory and non-linear analysis. Clarendon Press, Oxford, 2001.

[KO] Koskela, P., and J. Onninen: Mappings of finite distortion: Capacity and modulus inequalities. - J. Reine Angew. Math. 599, 2006, 1-26.

[MRV] Martio, O., S. Rickman, and J. VÄIsÄlä: Definitions for quasiregular mappings. Ann. Acad. Sci. Fenn. Ser. A I Math. 448, 1969, 1-40.

[MrSY] Martio, O., V. Ryazanov, U. Srebro, and E. Yakubov: Moduli in modern mapping theory. - Springer, New York, 2009.

[Maz] MaZ'YA, V.: Sobolev spaces. - Springer-Verlag, Berlin, 1985.

[Na] NÄKki, R.: Boundary behavior of quasiconformal mappings in $n$-space. - Ann. Acad. Sci. Fenn. Ser. A I Math. 484, 1970, 1-50.

[RR] RAdo, T., and P V. Reichelderfer: Continuous transformations in analysis. - SpringerVerlag, Berlin etc., 1955.

[Re] Reshetnyak, Yu. G.: Space mappings with bounded distortion. English transl. - Amer. Math. Soc., Providence, RI, 1989.

[Ri] Rickman, S.: Quasiregular mappings. - Springer-Verlag, Berlin etc., 1993.

[Sa] SAKs, S.: Theory of the integral. - Dover Publ. Inc., New York, 1964.

[Sev] Sevost'Yanov, E. A.: On the openess and discreteness of mappings with unbounded characteristic of quasiconormality. - Ukrainian Math. J. 63:8, 2012, 1298-1305.

[TY] Titus, C. J., and G.S. Young: The extension of interiority with some applications. Trans. Amer. Math. Soc. 103, 1962, 329-340.

[UV] Ukhlov, A.D., and S. K. Vodop'Yanov: Sobolev spaces and mappings with bounded $(P ; Q)$-distortion on Carnot groups. - Bull. Sci. Mat. 54:4, 2009, 349-370.

[Va] VÄıs̈̈LÄ, J.: Lectures on $n$-dimensional quasiconformal mappings. - Lecture Notes in Math. 229, Springer-Verlag, Berlin etc., 1971. 